BU-HEPP-03/02

CASPER-03/17

hep-ph/0310155

September 2003

\title{
Heterotic String Optical Unification
}

\author{
J. Perkins,${ }^{1 *}$ B. Dundee,${ }^{1 \dagger}$ R. Obousy,${ }^{1 \ddagger}$ E. Kasper,${ }^{1,3 \S}$ M. \\ Robinson, ${ }^{1,4}$ K. Stone, ${ }^{1,5 \|}$ \& G. Cleaver, ${ }^{1,2 * *}$ \\ 1 Center for Astrophysics, Space Physics 8 Engineering Research, \\ Dept. of Physics, PO Box 97316, Baylor University, \\ Waco, TX 76798-7316 \\ 2 Astro Particle Physics Group, Houston Advanced Research Center (HARC), \\ The Mitchell Campus, Woodlands, TX 77381 \\ 3 Department of Physics, Texas A $\&$ M University, \\ College Station, TX 77843-4242 \\ 4 Department of Physics, Auburn University, \\ Auburn, AL 36849 \\ 5 Department of Physics, East Tennessee State University, \\ Johnson City, TN, 37614
}

\footnotetext{
*John_Perkins@baylor.edu

${ }^{\dagger}$ Ben_Dundee@baylor.edu

‡Richard_K_Obousy@baylor.edu

§ekasper@physics.tamu.edu

ฯrobinmb@auburn.edu

" zkrs18@imail.etsu.edu

**Gerald_Cleaver@baylor.edu
} 


\begin{abstract}
The lower limit to string coupling unification in weakly coupled heterotic strings was shown by Kaplunovsky to be around $\Lambda_{H} \sim 5 \times 10^{17} \mathrm{GeV}$. In contrast, under the scenario of an intermediate scale desert, the $S U(3)_{C} \times$ $S U(2)_{L} \times U(1)_{Y}([321])$ Minimal Supersymmetric Standard Model (MSSM) unification scale is $\Lambda_{U} \sim 2.5 \times 10^{16} \mathrm{GeV}$. Optical unification was proposed by Giedt as a robust mechanism for relating these two scales. In this mechanism, intermediate scale MSSM-charged exotic particles effect running couplings like a diverging lens, always producing a "virtual" image of the string unification point between the string scale and the exotic particle mass scale. A heterotic string model that offers the potential to realize optical unification was recently constructed. This paper reports the initial results of an investigation into the possibility of optical unification realization in this model.

This paper is a product of the NSF REU program at Baylor University and is based on a talk presented at the Second International Conference on String Phenomenology, Durham, England, 29 July - 4 August, 2003.
\end{abstract}




\section{Review of Optical Unification}

In weakly coupled heterotic strings, the unification scale for running couplings has a lower limit of around $\Lambda_{H} \sim 5 \times 10^{17} \mathrm{GeV}[1]$. In contrast, the running of the couplings upward from measured values at $M_{Z^{\circ}}$ predict Minimal Supersymmetric Standard Model (MSSM) $S U(3)_{C} \times S U(2)_{L} \times U(1)_{Y}([321])$ gauge coupling unification at $\Lambda_{U} \sim 2.5 \times 10^{16} \mathrm{GeV}$ when an intermediate scale desert is assumed [2]. One resolution is a grand unified theory (GUT) between the MSSM and string scales. However, with the exception of flipped $S U(5)$ [3] (or partial GUTs such as the PatiSalam $\left.S U(4)_{C} \times S U(2)_{L} \times S U(2)_{R}[4,5]\right)$, string GUTs cannot be generated by levelone Kač-Moody algebras (since they lack the required adjoint higgs and/or higher dimensional scalar representations) and models based on higher level Kač-Moody algebras vastly prefer even numbers of generations [6, 7, 8]. Alternately, strong coupling effects of $M$-theory can lower $\Lambda_{H}$ down to $\Lambda_{U}$ [9]. On the other hand, intermediate scale $\Lambda_{I}<\Lambda_{U}$ MSSM-charged exotics could shift the MSSM unification scale upward to the string scale [10].

The near ubiquitous appearance of MSSM-charged exotics in heterotic string models adds weight to the third proposal. However, most intermediate scale MSSMcharged exotic solutions might be viewed as accidental. While existence of the string unification scale would clearly be stable under shifts in the masses of the exotics, the prediction of an apparent lower-scale MSSM unification when MSSM-charged exotics are ignored would generally be unstable under these mass shifts. On the other hand, a set of intermediate scale MSSM-charged exotic particles satisfying "optical unification" constraints provide a robust method for stablizing apparent MSSM unification under such shifts [11]. With optical unification, a shift of the intermediate scale $\Lambda_{I}$ produces a shift in $\Lambda_{U}$, rather than the disappearance of $\Lambda_{U}$. This effect is parallel to a diverging lens always producing a virtual image between itself and a real object, independent of the position of the lens or real object. Hence, the choice of appellation for this mechanism.

Successful optical unification requires three things [11]. First, the effective level of the hypercharge generator must be the standard

$$
k_{Y}=\frac{5}{3}
$$

(1.1) is a strong constraint on string-derived [321] models, for the vast majority have non-standard hypercharge levels. Only select classes of models, such as the free fermionic [12] NAHE-based [13] class, can yield $k_{Y}=\frac{5}{3}$.

Second, optical unification imposes the relationship

$$
\delta b_{2}=\frac{7}{12} \delta b_{3}+\frac{1}{4} \delta b_{Y}
$$

between the exotic particle contributions $\delta b_{3}, \delta b_{2}$, and $\delta b_{1}$ to the [321] beta function coefficients. Each $S U(3)_{C}$ exotic triplet or anti-triplet contributes $\frac{1}{2}$ to $\delta b_{3}$; each 
$S U(2)_{C}$ exotic doublet contributes $\frac{1}{2}$ to $\delta b_{2}$. With the hypercharge of a MSSM quark doublet normalized to $\frac{1}{6}$, the contribution to $\delta b_{Y}$ from an individual particle with hypercharge $Q_{Y}$ is $Q_{Y}^{2}$. $\delta b_{3}>\delta b_{2}$ is required to keep the virtual unification scale below the string scale. Combining all of this with (1.2) imposes

$$
\delta b_{3}>\delta b_{2} \geq \frac{7}{12} \delta b_{3},
$$

since $\delta b_{Y} \geq 0$.

To acquire intermediate scale mass, the exotic triplets and anti-triplets must be equal in number. Similarly, there must be an even number of exotic doublets. Hence, $\delta b_{3}$ and $\delta b_{2}$ must be integer. The simplest solution to (1.2) and (1.3) is a set of three exotic triplet/anti-triplet pairs and two pairs of doublets. One pair of doublets can carry $Q_{Y}= \pm \frac{1}{2}$, while the remaining exotics carry no hypercharge [11]. Alternately, if the doublets carry too little hypercharge, some exotic $S U(3)_{C} \times S U(2)_{L}$ singlets could make up the hypercharge deficit. The next simplest solution requires four triplet/anti-triplet pairs and three pairs of doublets that yield $\delta b_{Y}=2 \frac{2}{3}$ either as a set, or with the assistance of additional non-Abelian singlets. For more than four triplet/anti-triplet pairs, (1.2) and (1.3) allow varying numbers of pairs of doublets.

\section{Flat Directions of String Models}

Quasi-realistic heterotic string models generically contain an anomalous $U(1)_{A}$ (for which $\operatorname{Tr} Q^{(A)} \neq 0$ ) [14]. The Green-Schwarz-Dine-Seiberg-Witten mechanism [15] breaks the anomalous U(1), and in the process generates a Fayet-Iliopoulos (FI) term,

$$
\epsilon \equiv \frac{g_{s}^{2} M_{P}^{2}}{192 \pi^{2}} \operatorname{Tr} Q^{(A)},
$$

in the associated $D$-term. The FI term breaks supersymmetry near the Planck scale and destabilize the string vacuum, unless it is cancelled by scalar vacuum expectation values (VEVs),

$$
\left\langle D_{A}\right\rangle=\sum_{m} Q_{m}^{(A)}\left|\left\langle\varphi_{m}\right\rangle\right|^{2}+\epsilon=0 .
$$

Thus, an effect of the anomalous $U(1)_{A}$ is to induce a non-perturbatively chosen flat direction of VEVs. Since the fields taking on the VEVs typically carry additional non-anomalous charges, a non-trivial set of constraints is imposed on the VEVs. The VEVs must maintain $D$-flatness for each non-anomalous gauge symmetry. For non-anomalous Abelian symmetries it is required that,

$$
\left\langle D_{i}\right\rangle=\sum_{m} Q_{m}^{(i)}\left|\left\langle\varphi_{m}\right\rangle\right|^{2}=0,
$$


while for non-Abelian symmetries,

$$
\left\langle D_{a}^{\alpha}\right\rangle=\sum_{m}\left\langle\varphi_{m}^{\dagger} T_{a}^{\alpha} \varphi_{m}\right\rangle=0
$$

with $T_{a}^{\alpha}$ a matrix generator of the non-Abelian symmetry. The scalar VEVs will in general break some, or all, of the additional gauge symmetries spontaneously.

To insure a supersymmetric vacuum $F$-flatness,

$$
\left\langle F_{m}\right\rangle \equiv\left\langle\frac{\partial W}{\partial \Phi_{m}}\right\rangle=0,
$$

must also be maintained for each superfield $\Phi_{m}$ (containing a scalar field $\varphi_{m}$ and chiral spin- $\frac{1}{2}$ superpartner $\psi_{m}$ ) appearing in the superpotential $W$.

A typical string model contains a moduli space of perturbative solutions to the $D$ - and $F$-flatness constraints, which are supersymmetric and degenerate in energy [16]. Much of the study of the phenomenology of superstring models involves the analysis and classification of these flat directions. The methods for this analysis in string models have been systematized in recent years $[17,18,19,5]$.

\section{$3 \quad$ String Model with Optical Unification Potential}

The possibility of optical unification within the context of free fermionic heterotic strings was investigated recently $[20,21]$. By altering some of the GSO projection coefficients in the models originally presented in [22], but keeping the same free fermionic boundary vectors, a model with a set of $S U(3)_{C}$ exotic triplet/anti-triplet pairs, $S U(2)_{L}$ exotic doublets, and non-Abelian singlets satisfying optical unification requirements was constructed. Specifically, the model contains three pairs of exotic triplet/anti-triplets with $Q_{Y}= \pm \frac{1}{3}$, one pair of exotic triplet/anti-triplets with $Q_{Y}=$ $\pm \frac{1}{6}$, three pairs of exotic doublets with $Q_{Y}=0$, and seven pairs of singlets with $Q_{Y}= \pm \frac{1}{2}$. The model also contains four copies of higgs doublet pairs. (See Table 1 below. The complete set of states are given in Ref. [20].) Optical unification requires that all four triplet/anti-triplet pairs, all three doublet pairs, and one pair of of exotic singlets with hypercharge take on an intermediate scale mass. The remaining six pairs of exotic singlets with hypercharge must take on masses above the MSSM unification scale, as must three out of four orthogonal combinations of the higgs.

The proposed optical unification model contains an anomalous $U(1)_{A}$. Thus some of its scalar fields will necessarily receive FI-scale VEVs. We have begun a systematic search for flat direction VEVs that will induce FI-scale masses for the six extra pairs of exotic $Q_{Y}= \pm \frac{1}{2}$ singlets and the three extra higgs, while keeping the four exotic triplet/anti-triplets pairs, three exotic doublet pairs, and one exotic $Q_{Y}= \pm \frac{1}{2}$ singlent pair FI-scale massless. The first step in our sytematic search for $D$ - and $F$-flat directions was to generate a basis set of $D$-flat directions. Routines have been developed for this using a singular value decomposition approach [18, 23]. 
In our initial investigation only singlet fields with $Q_{Y}=0$ were allowed VEVs. The model contains 27 such fields: the three uncharged moduli $\Phi_{i=1,2,3} ; \Phi_{12}, \Phi_{23}, \Phi_{31}$, and their respective complex conjugate fields $\bar{\Phi}_{12}, \bar{\Phi}_{23}, \bar{\Phi}_{31}$; and $S_{j=1 \text { to } 9}$ and their

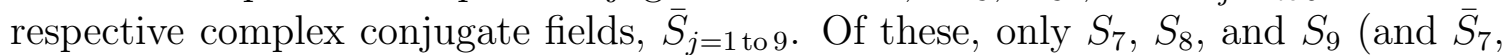
$\bar{S}_{8}$, and $\bar{S}_{9}$ ) carry anomalous charge. All $D$-flat directions containing only VEVs of these 27 singlet fields can be generated from a basis set of nine elements, denoted $\mathcal{D}_{i=1 \text { to } 9}$, given in Table 3 below. Only $\mathcal{D}_{1}$, corresponds to a net negative anomalous charge, while all of the other eight correspond to no anomalous charge. Since the FI term has positive sign, any $D$-flat direction must contain $\mathcal{D}_{1}$ and can contain any number of the $\mathcal{D}_{i>1}$.

We applied stringent $F$-flatness constraints to all $D$-flat directions formed as linear combinations of the basis elements $\mathcal{D}_{i}$, with coefficients ranging from 1 to 50 for $\mathcal{D}_{1}$ and $\mathcal{D}_{i \geq 7}$, and ranging from -50 to 50 for $\mathcal{D}_{1<i<7}$. Since the $\Phi_{12}, \Phi_{23}, \Phi_{31}$, and $S_{i}$ fields have vector-like partner (complex conjugate) fields, a negative coefficient implies that the relevant vector partners take on the net VEVs. Stringent $F$-flat directions are those for which $F$-flatness does not require cancellation between two or more terms in a given $F$-term. This implies that at least two fields in a given $F$-term do not take on VEVs. We found that only one class of $D$-flat directions was stringently $F$-flat to all order. The FI-scale field VEVs for this class are:

$$
<\bar{S}_{7} \bar{S}_{8} S_{9}><\bar{\Phi}_{12} \text { and/or } \bar{\Phi}_{31} \text { and/or } \Phi_{2}>
$$

That is, the fields $\bar{S}_{7}, \bar{S}_{8}$, and $S_{9}$ must take on VEVs, while VEVs are optional for $\bar{\Phi}_{12}, \bar{\Phi}_{31}$, and $\Phi_{2}$. The relative ratios of the squares of the norms of the VEVs vary for each option but are all of $\mathcal{O}(1$ to 10$)$. All other $D$-flat directions generated break $F$-flatness at third to fifth order in the superpotential.

For the (3.1) class of flat directions none of the seven pairs of singlets with $Q_{Y}= \pm \frac{1}{2}$ receive mass. In constrast, six pairs must receive FI scale mass for optical unification. The three pairs of exotic doublets remain FI-scale massless as desired, as do the four pairs of exotic triplet/anti-triplets, unless $\Phi_{2}$ receives a VEV. In the latter event, one exotic triplet/anti-triplet pair receives FI-scale mass from a third order term, $D_{4} \bar{D}_{4}<\Phi_{2}>$, and another from a fifth order term, $D_{1} \bar{D}_{2}<\Phi_{2} \bar{S}_{8} S_{9}>$. The VEV of $S_{9}$ also results in one pair of higgs receiving FI-scale mass from a third order superpotential term. If $\bar{\Phi}_{12}$ or $\bar{\Phi}_{31}$ receive a VEV, a second pair of higgs also receives similar mass.

The singlet flat-direction class (3.1) is insufficient because of its lack of related mass terms for the six extra pairs of singlets. Since all other singlet $D$-flat directions examined by us lose $F$-flatness before sixth order, non-Abelian flat directions appear necessary for realization of optical unification in our model. Thus we have begun to investigate phenomenologies of flat directions containing VEVs of the model's hidden sector fields. The model contains four pairs of $S U(5)_{H} 5 / \overline{5}$ fields, and four pairs of $S U(3)_{H} 3 / \overline{3}$ fields. We have found that all basis elements of non-Abelian $D$-flat directions make a positive contribution to the anomalous $D$-term. Thus, any $D$-flat 
direction with non-Abelian fields must contain the singlet direction $\mathcal{D}_{1}$ (the only basis element with a net negative contribution) with a sufficiently large positive coefficient. The results of our non-Abelian flat direction study will appear in Ref. [24]. We will also be undertaking a systematic search for additional heterotic string models with optical unification potential.

Optical unification offers a phenomenologically appealing resolution to the factor of twenty difference between the apparent $\Lambda_{U} \sim 2.5 \times 10^{16} \mathrm{GeV}$ scale for MSSM unification scale, assuming an intermediate scale desert, and the lower bound of $\Lambda_{H} \sim 5 \times 10^{17} \mathrm{GeV}$ for coupling unification in weakly coupled heterotic strings. However, it is still unclear whether optical unification can, in fact, be realized in string models. Whether this is possible for NAHE-based free fermionic models, in particular, should be determined within the next year.

\section{Acknowledgments}

This paper is a product of the 2003 NSF REU program sponsored by the Center for Astrophysics, Space Physics, and Engineering Research (CASPER) at Baylor University. Research funding for Eric Kasper, Matthew Robinson, and Kristin Stone was provided by NSF grant no. 0097386. G.C. thanks Alon Faraggi and Dimitri Nanopoulos for numerous discussions of NAHE-based models and thanks Joel Giedt for discussions regarding optical unification. 


\section{References}

[1] V.S. Kaplunovsky, Nucl. Phys. B307 (1988) 145; Erratum Nucl. Phys. B382 (1992) 436.

[2] J. Ellis, S. Kelley and D.V. Nanopoulos, Phys. Lett. B249 (1990) 441;

U. Amaldi, W. de Boer and F. Fürstenau, Phys. Lett. B260 (1991) 447;

P. Langacker and M. Luo, Phys. Rev. D44 (1991) 817.

[3] I. Antoniadis, J. Ellis, J. Hagelin, and D.V. Nanopoulos, Phys. Lett. B231 (1989) 65

I. Antoniadis, J. Ellis, R. Lacaze, D.V. Nanopoulos, Phys. Lett. B268 (1991) 188 ;

J. Lopez, D.V. Nanopoulos and K. Yuan, Nucl. Phys. B399 (1993) 654;

J. Lopez and D.V. Nanopoulos, [hep-ph/9511266];

A. Dedes, C. Panagiotakopoulos, K. Tamvakis, Phys. Rev. D57 (1998) 5493, [hep-ph/9710563];

J. Ellis, G. Leontaris, S. Lola, and D.V. Nanopoulos, Phys. Lett. B425 (1998) 86, [hep-ph/9711476]; K.T. Mahanthappa, Phys. Lett. B441 (1998) 178, [hep$\mathrm{ph} / 9807231]$

J. Ellis, G. Leontaris, and J. Rizos, Phys. Lett. B464 (1999) 62, [hep$\mathrm{ph} / 9907476]$;

G. Cleaver, J. Ellis, and D.V. Nanopoulos, Nucl. Phys. B600 (2001) 315, [hep$\mathrm{ph} / 0009338]$;

A.E. Faraggi, R. Garavuso, and J.M. Isidro, [hep-ph/0204080];

J. Ellis and D.V. Nanopoulos, Phys. Lett. B550 (2002) 99, [hep-ph/0205336];

J. Ellis, P. Kanti, and D.V. Nanopoulos, Nucl. Phys. B647 (2002) 235; [hep$\mathrm{ph} / 0206087$.

[4] J. Pati and R.N. Mohapatra, Phys. Rev. D11 (1975) 2558;

D. Chang, R.N. Mohapatra, M.K. Parida, Phys. Rev. D30 (1984) 1052;

I. Antoniadis, G.K. Leontaris, and J. Rizos, Phys. Lett. B245 (1990) 161;

G.K. Leontaris, and J. Rizos, Nucl. Phys. B554 (1999) 3, [hep-th/9901098];

F. Astorga, AIP Conf. Proc. 478 (1999) 277;

Y. Mimura, S. Nandi, Phys. Lett. B538 (2002) 406, [hep-ph/0203126].

[5] G.B. Cleaver, A.E. Faraggi and C. Savage, Phys. Rev. D63 (2001) 066001, [hep$\mathrm{ph} / 0006331$;

G.B. Cleaver, D. Clements, A.E. Faraggi, Phys. Rev. D65 (2002) 106003, [hep$\mathrm{ph} / 0106060]$.

[6] G. Cleaver, [hep-th/9409096]; [hep-th/9506006]; [hep-th/9604183].

[7] S. Chaudhuri, S. Chung, J. Lykken, [hep-ph/9405374];

S. Chaudhuri, S.W. Chung, G. Hockney, J.D. Lykken, Nucl. Phys. B456 (1995) 
89, [hep-ph/9501361];

S. Chaudhuri, G. Hockney, J.D. Lykken, Nucl. Phys. B459 (1996) 357, [hepth/9510241].

[8] Z. Kakushadze and S.H.-H. Tye, Phys. Rev. D54 (1996) 7520, [hep-th/9607138]; Z. Kakushadze, G. Shiu, and S.H.-H. Tye, Phys. Rev. D54 (1996) 7545, [hepth/9607137]; Phys. Lett. B392 (1997) 335, [hep-th/9609027]; Phys. Rev. D55 (1997) 7878, [hep-th/9610106].

[9] E. Witten, Nucl. Phys. B471 (1996) 135, [hep-th/9602070].

[10] S. Chang, C. Coriano, and A. Faraggi, Phys. Lett. B397 (1997) 76, [hep$\mathrm{ph} / 9603272]$.

[11] J. Giedt, Mod. Phys. Lett. A18 (2003) 1625, [hep-ph/0205224].

[12] I. Antoniadis, C. Bachas, and C. Kounnas, Nucl. Phys. B289 (1987) 87;

H. Kawai, D.C. Lewellen, and S.H.-H. Tye, Nucl. Phys. B288 (1987) 1.

[13] A.E. Faraggi and D.V. Nanopoulos, Phys. Rev. D48 (1993) 3288;

A.E. Faraggi, [hep-th/9511093]; [hep-th/9708112].

[14] A. Schellekens, in Superstring Construction, (North-Holland, New York, 1989) p. 2 ;

A. Schellekens and N. Warner, Nucl. Phys. B313 (1989) 41;

T. Kobayashi and H. Nakano, Nucl. Phys. B496 (1997) 103, [hep-th/9612066];

G. Cleaver and A.E. Faraggi, Int. J. Mod. Phys. A14 (1999) 2335, [hep$\mathrm{ph} / 9711339]$.

[15] M. Green and J. Schwarz, Phys. Lett. B149 (1984) 117;

M. Dine, N. Seiberg and E. Witten, Nucl. Phys. B289 (1987) 589;

J. Atick, L. Dixon and A. Sen, Nucl. Phys. B292 (1987) 109.

[16] A. Font, L. Ibanez, H. Nilles, and F. Quevedo, Nucl. Phys. B307 (1988) 109, Erratum Nucl. Phys. B310 (1988) 764;

J.A. Casas, E.K. Katehou and C. Munoz, Nucl. Phys. B317 (1989) 171;

M.K. Gaillard and J. Giedt, Phys. Lett. B479 (2000) 308, [hep-ph/0001219].

[17] G. Cleaver, M. Cvetič, J. Espinosa, L. Everett, and P. Langacker, Nucl. Phys. B525 (1998) 3, [hep-th/9711178]; Nucl. Phys. B545 (1999) 47, [hepth/9805133];

G. Cleaver, M. Cvetič, J. Espinosa, L. Everett, P. Langacker, and J. Wang, Phys. Rev. D59 (1999) 055005, [hep-th/9807479]; Phys. Rev. D59 (1999) 115003, [hepth/9811355]. 
[18] G.B. Cleaver, A.E. Faraggi and D.V. Nanopoulos, Phys. Lett. B445 (1999) 135, [hep-ph/9811427]; Int. J. Mod. Phys. A16 (2001) 425, [hep-ph/9904301];

G.B. Cleaver, A.E. Faraggi, D.V. Nanopoulos, and J.W. Walker, Nucl. Phys. B593 (2001) 471, [hep-ph/9910230]; Mod. Phys. Lett. A15 (2000) 1191, [hepph/0002060]; Nucl. Phys. B620 (2002) 259, [hep-ph/0104091];

G. Cleaver, [hep-ph/9901203]; Int. J. Mod. Phys. A16S1C (2001) 949, [hepph/0011020]; [hep-ph/0003208]; [hep-ph/0301037].

[19] G. Cleaver, A.E. Faraggi, and T. ter Veldhuis, Int. J. Mod. Phys. A16 (2001) 3565, [hep-ph/0002292].

[20] G. Cleaver, V. Desai, H. Hanson, J. Perkins, D. Robbins, and S. Shields, Phys. Rev. D67 (2003) 026009, [hep-ph/0209050].

[21] G. Cleaver, [hep-ph/0210093].

[22] A.E. Faraggi, Phys. Lett. B274 (1992) 47; Phys. Lett. B302 (1993) 202, [hepph/9301268]; Phys. Lett. B339 (1994) 223, [hep-ph/9408333].

[23] G. Cleaver, [hep-ph/0003208].

[24] J. Perkins, B. Dundee, R. Obousy, E. Kasper, M. Robinson, K. Stone, \& G. Cleaver, Phenomenological Studies of Heterotic String Optical Unification. To appear. 
Table 1: Optical Unification Higgs \& MSSM Exotics

\begin{tabular}{|c|c|c|c|c|c|c|c|c|c|c|c|c|}
\hline$F$ & $\begin{array}{c}\left(S U(3)_{C},\right. \\
\left.S U(2)_{L}\right)\end{array}$ & $Q_{Y}$ & $Q_{Z^{\prime}}$ & $\overline{Q_{A}}$ & $\overline{Q_{1}}$ & $\overline{Q_{2}}$ & $\overline{Q 3}$ & $\overline{Q_{4}}$ & $Q_{5}$ & $\begin{array}{l}\left(S U(5)_{H},\right. \\
\left.S U(3)_{H}\right)\end{array}$ & $Q_{6}$ & $Q_{7}$ \\
\hline$h_{1}$ & $(1,2)$ & $-1 / 2$ & $1 / 2$ & 1 & -1 & 1 & 0 & 0 & 0 & $(1,1)$ & 0 & 0 \\
\hline$h_{2}$ & $(1,2)$ & $-1 / 2$ & $1 / 2$ & 1 & 1 & 1 & 0 & 0 & 0 & $(1,1)$ & 0 & 0 \\
\hline$h_{3}$ & $(1,2)$ & $-1 / 2$ & $1 / 2$ & 1 & 0 & -2 & 0 & 0 & 0 & $(1,1)$ & 0 & 0 \\
\hline$h_{4}$ & $(1,2)$ & $-1 / 2$ & 0 & $-1 / 4$ & $-1 / 2$ & $1 / 2$ & 0 & 0 & 0 & $(1,1)$ & 2 & 0 \\
\hline $\bar{h}_{1}$ & $(1,2)$ & $1 / 2$ & $-1 / 2$ & -1 & 1 & -1 & 0 & 0 & 0 & $(1,1)$ & 0 & 0 \\
\hline $\bar{h}_{2}$ & $(1,2)$ & $1 / 2$ & $-1 / 2$ & -1 & -1 & -1 & 0 & 0 & 0 & $(1,1)$ & 0 & 0 \\
\hline $\bar{h}_{3}$ & $(1,2)$ & $1 / 2$ & $-1 / 2$ & -1 & 0 & 2 & 0 & 0 & 0 & $(1,1)$ & 0 & 0 \\
\hline $\bar{h}_{4}$ & $(1,2)$ & $1 / 2$ & 0 & $1 / 4$ & $1 / 2$ & $-1 / 2$ & 0 & 0 & 0 & $(1,1)$ & -2 & 0 \\
\hline$\overline{D_{1}}$ & $(3,1)$ & $-1 / 3$ & $-1 / 3$ & 1 & 0 & 1 & 0 & 0 & 0 & $(1,1)$ & 0 & 0 \\
\hline$D_{2}$ & $(3,1)$ & $-1 / 3$ & $-1 / 3$ & -1 & 0 & -1 & 0 & 0 & 0 & $(1,1)$ & 0 & 0 \\
\hline$D_{3}$ & $(3,1)$ & $-1 / 3$ & $1 / 6$ & $1 / 4$ & $-1 / 2$ & $-1 / 2$ & 0 & 0 & 0 & $(1,1)$ & -2 & 0 \\
\hline$D_{4}$ & $(3,1)$ & $1 / 6$ & $1 / 6$ & 0 & 0 & 0 & $1 / 2$ & $1 / 2$ & $1 / 2$ & $(1,1)$ & $1 / 2$ & $-15 / 2$ \\
\hline $\bar{D}_{1}$ & $(\overline{3}, 1)$ & $1 / 3$ & $1 / 3$ & -1 & 0 & -1 & 0 & 0 & 0 & $(1,1)$ & 0 & 0 \\
\hline $\bar{D}_{2}$ & $(\overline{3}, 1)$ & $1 / 3$ & $1 / 3$ & 1 & 0 & 1 & 0 & 0 & 0 & $(1,1)$ & 0 & 0 \\
\hline $\bar{D}_{3}$ & $(\overline{3}, 1)$ & $1 / 3$ & $1 / 6$ & $-1 / 4$ & $1 / 2$ & $1 / 2$ & 0 & 0 & 0 & $(1,1)$ & 2 & 0 \\
\hline $\bar{D}_{4}$ & $(\overline{3}, 1)$ & $-1 / 6$ & $-1 / 6$ & 0 & 0 & 0 & $-1 / 2$ & $-1 / 2$ & $-1 / 2$ & $(1,1)$ & $-1 / 2$ & $15 / 2$ \\
\hline$X_{1}$ & $(1,2)$ & 0 & 0 & $1 / 2$ & $-1 / 2$ & $1 / 2$ & $1 / 2$ & 0 & $1 / 2$ & $(1,1)$ & $-1 / 2$ & $15 / 2$ \\
\hline$X_{2}$ & $(1,2)$ & 0 & 0 & $1 / 2$ & $1 / 2$ & $1 / 2$ & 0 & $-1 / 2$ & $1 / 2$ & $(1,1)$ & $-1 / 2$ & $15 / 2$ \\
\hline$X_{3}$ & $(1,2)$ & 0 & 0 & $1 / 2$ & 0 & -1 & $1 / 2$ & $-1 / 2$ & 0 & $(1,1)$ & $-1 / 2$ & $15 / 2$ \\
\hline $\bar{X}_{1}$ & $(1,2)$ & 0 & 0 & $-1 / 2$ & $1 / 2$ & $-1 / 2$ & $1 / 2$ & 0 & $1 / 2$ & $(1,1)$ & $1 / 2$ & $-15 / 2$ \\
\hline $\bar{X}_{2}$ & $(1,2)$ & 0 & 0 & $-1 / 2$ & $-1 / 2$ & $-1 / 2$ & 0 & $-1 / 2$ & $1 / 2$ & $(1,1)$ & $1 / 2$ & $-15 / 2$ \\
\hline $\bar{X}_{3}$ & $(1,2)$ & 0 & 0 & $-1 / 2$ & 0 & 1 & $1 / 2$ & $-1 / 2$ & 0 & $(1,1)$ & $1 / 2$ & $-15 / 2$ \\
\hline$\overline{A_{1}}$ & $(1,1)$ & $1 / 2$ & $1 / 2$ & 0 & 0 & 0 & $1 / 2$ & $1 / 2$ & $-1 / 2$ & $(1,1)$ & $-1 / 2$ & $15 / 2$ \\
\hline$A_{2}$ & $(1,1)$ & $-1 / 2$ & $1 / 2$ & $-1 / 2$ & $-1 / 2$ & $-1 / 2$ & 0 & $1 / 2$ & $-1 / 2$ & $(1,1)$ & $-1 / 2$ & $15 / 2$ \\
\hline$A_{3}$ & $(1,1)$ & $-1 / 2$ & $1 / 2$ & $-1 / 2$ & 0 & 1 & $-1 / 2$ & $1 / 2$ & 0 & $(1,1)$ & $-1 / 2$ & $15 / 2$ \\
\hline$A_{4}$ & $(1,1)$ & $-1 / 2$ & $1 / 2$ & $-1 / 2$ & $1 / 2$ & $-1 / 2$ & $-1 / 2$ & 0 & $-1 / 2$ & $(1,1)$ & $-1 / 2$ & $15 / 2$ \\
\hline$A_{5}$ & $(1,1)$ & $1 / 2$ & $-1 / 2$ & $-1 / 2$ & $-1 / 2$ & $-1 / 2$ & 0 & $1 / 2$ & $-1 / 2$ & $(1,1)$ & $-1 / 2$ & $15 / 2$ \\
\hline$A_{6}$ & $(1,1)$ & $1 / 2$ & $-1 / 2$ & $-1 / 2$ & 0 & 1 & $-1 / 2$ & $1 / 2$ & 0 & $(1,1)$ & $-1 / 2$ & $15 / 2$ \\
\hline$A_{7}$ & $(1,1)$ & $1 / 2$ & $-1 / 2$ & $-1 / 2$ & $1 / 2$ & $-1 / 2$ & $-1 / 2$ & 0 & $-1 / 2$ & $(1,1)$ & $-1 / 2$ & $15 / 2$ \\
\hline $\bar{A}_{1}$ & $(1,1)$ & $-1 / 2$ & $-1 / 2$ & 0 & 0 & 0 & $-1 / 2$ & $-1 / 2$ & $1 / 2$ & $(1,1)$ & $1 / 2$ & $-15 / 2$ \\
\hline $\bar{A}_{2}$ & $(1,1)$ & $1 / 2$ & $-1 / 2$ & $1 / 2$ & $1 / 2$ & $1 / 2$ & 0 & $1 / 2$ & $-1 / 2$ & $(1,1)$ & $1 / 2$ & $-15 / 2$ \\
\hline $\bar{A}_{3}$ & $(1,1)$ & $1 / 2$ & $-1 / 2$ & $1 / 2$ & 0 & -1 & $-1 / 2$ & $1 / 2$ & 0 & $(1,1)$ & $1 / 2$ & $-15 / 2$ \\
\hline $\bar{A}_{4}$ & $(1,1)$ & $1 / 2$ & $-1 / 2$ & $1 / 2$ & $-1 / 2$ & $1 / 2$ & $-1 / 2$ & 0 & $-1 / 2$ & $(1,1)$ & $1 / 2$ & $-15 / 2$ \\
\hline $\bar{A}_{5}$ & $(1,1)$ & $-1 / 2$ & $1 / 2$ & $1 / 2$ & $1 / 2$ & $1 / 2$ & 0 & $1 / 2$ & $-1 / 2$ & $(1,1)$ & $1 / 2$ & $-15 / 2$ \\
\hline $\bar{A}_{6}$ & $(1,1)$ & $-1 / 2$ & $1 / 2$ & $1 / 2$ & 0 & -1 & $-1 / 2$ & $1 / 2$ & 0 & $(1,1)$ & $1 / 2$ & $-15 / 2$ \\
\hline $\bar{A}_{7}$ & $(1,1)$ & $-1 / 2$ & $1 / 2$ & $1 / 2$ & $-1 / 2$ & $1 / 2$ & $-1 / 2$ & 0 & $-1 / 2$ & $(1,1)$ & $1 / 2$ & $-15 / 2$ \\
\hline
\end{tabular}


Table 2: Optical Unification Singlets with $Q_{Y}=0$

\begin{tabular}{|l||c|cccccccc|c|cc|}
\hline$F$ & $\left(S U(3)_{C}\right.$, & $Q_{Y}$ & $Q_{Z^{\prime}}$ & $Q_{A}$ & $Q_{1}$ & $Q_{2}$ & $Q_{3}$ & $Q_{4}$ & $Q_{5}$ & $\left(S U(5)_{H}\right.$, & $Q_{6}$ & $Q_{7}$ \\
& $\left.S U(2)_{L}\right)$ & & & & & & & & & $\left.S U(3)_{H}\right)$ & & \\
\hline$\Phi_{1}$ & $(1,1)$ & 0 & 0 & 0 & 0 & 0 & 0 & 0 & 0 & $(1,1)$ & 0 & 0 \\
$\Phi_{2}$ & $(1,1)$ & 0 & 0 & 0 & 0 & 0 & 0 & 0 & 0 & $(1,1)$ & 0 & 0 \\
$\Phi_{3}$ & $(1,1)$ & 0 & 0 & 0 & 0 & 0 & 0 & 0 & 0 & $(1,1)$ & 0 & 0 \\
$\Phi_{12}$ & $(1,1)$ & 0 & 0 & 0 & -2 & 0 & 0 & 0 & 0 & $(1,1)$ & 0 & 0 \\
$\Phi_{23}$ & $(1,1)$ & 0 & 0 & 0 & 1 & -3 & 0 & 0 & 0 & $(1,1)$ & 0 & 0 \\
$\Phi_{31}$ & $(1,1)$ & 0 & 0 & 0 & -1 & -3 & 0 & 0 & 0 & $(1,1)$ & 0 & 0 \\
$\bar{\Phi}_{12}$ & $(1,1)$ & 0 & 0 & 0 & 2 & 0 & 0 & 0 & 0 & $(1,1)$ & 0 & 0 \\
$\bar{\Phi}_{23}$ & $(1,1)$ & 0 & 0 & 0 & -1 & 3 & 0 & 0 & 0 & $(1,1)$ & 0 & 0 \\
$\bar{\Phi}_{31}$ & $(1,1)$ & 0 & 0 & 0 & 1 & 3 & 0 & 0 & 0 & $(1,1)$ & 0 & 0 \\
$S_{1}$ & $(1,1)$ & 0 & 0 & 0 & -1 & 0 & -1 & 0 & 0 & $(1,1)$ & 0 & 0 \\
$S_{2}$ & $(1,1)$ & 0 & 0 & 0 & -1 & 0 & 1 & 0 & 0 & $(1,1)$ & 0 & 0 \\
$S_{3}$ & $(1,1)$ & 0 & 0 & 0 & -1 & 0 & 0 & -1 & 0 & $(1,1)$ & 0 & 0 \\
$S_{4}$ & $(1,1)$ & 0 & 0 & 0 & -1 & 0 & 0 & 1 & 0 & $(1,1)$ & 0 & 0 \\
$S_{5}$ & $(1,1)$ & 0 & 0 & 0 & -1 & 0 & 0 & 0 & -1 & $(1,1)$ & 0 & 0 \\
$S_{6}$ & $(1,1)$ & 0 & 0 & 0 & -1 & 0 & 0 & 0 & 1 & $(1,1)$ & 0 & 0 \\
$S_{7}$ & $(1,1)$ & 0 & $1 / 2$ & $3 / 4$ & $-1 / 2$ & $-3 / 2$ & 0 & 0 & 0 & $(1,1)$ & 2 & 0 \\
$S_{8}$ & $(1,1)$ & 0 & $1 / 2$ & $3 / 4$ & $1 / 2$ & $3 / 2$ & 0 & 0 & 0 & $(1,1)$ & 2 & 0 \\
$S_{9}$ & $(1,1)$ & 0 & $1 / 2$ & $-5 / 4$ & $1 / 2$ & $-1 / 2$ & 0 & 0 & 0 & $(1,1)$ & 2 & 0 \\
$\bar{S}_{1}$ & $(1,1)$ & 0 & 0 & 0 & 1 & 0 & 1 & 0 & 0 & $(1,1)$ & 0 & 0 \\
$\bar{S}_{2}$ & $(1,1)$ & 0 & 0 & 0 & 1 & 0 & -1 & 0 & 0 & $(1,1)$ & 0 & 0 \\
$\bar{S}_{3}$ & $(1,1)$ & 0 & 0 & 0 & 1 & 0 & 0 & 1 & 0 & $(1,1)$ & 0 & 0 \\
$\bar{S}_{4}$ & $(1,1)$ & 0 & 0 & 0 & 1 & 0 & 0 & -1 & 0 & $(1,1)$ & 0 & 0 \\
$\bar{S}_{5}$ & $(1,1)$ & 0 & 0 & 0 & 1 & 0 & 0 & 0 & 1 & $(1,1)$ & 0 & 0 \\
$\bar{S}_{6}$ & $(1,1)$ & 0 & 0 & 0 & 1 & 0 & 0 & 0 & -1 & $(1,1)$ & 0 & 0 \\
$\bar{S}_{7}$ & $(1,1)$ & 0 & $-1 / 2$ & $-3 / 4$ & $1 / 2$ & $3 / 2$ & 0 & 0 & 0 & $(1,1)$ & -2 & 0 \\
$\bar{S}_{8}$ & $(1,1)$ & 0 & $-1 / 2$ & $-3 / 4$ & $-1 / 2$ & $-3 / 2$ & 0 & 0 & 0 & $(1,1)$ & -2 & 0 \\
$\bar{S}_{9}$ & $(1,1)$ & 0 & $-1 / 2$ & $5 / 4$ & $-1 / 2$ & $1 / 2$ & 0 & 0 & 0 & $(1,1)$ & -2 & 0 \\
\hline
\end{tabular}


Table 3: $D$-Flat Basis Elements. The first entry in a given row denotes the $D$-flat basis element, the second entry its anomalous charge, and the remaining entries, the ratios of the squares of the norms of the field VEVs defining the basis element.

\begin{tabular}{|l||r|rrrrrrrrrrrrrrr|}
\hline Dir & $Q^{(A)}$ & $\Phi_{12}$ & $\Phi_{23}$ & $\Phi_{31}$ & $S_{1}$ & $S_{2}$ & $S_{3}$ & $S_{4}$ & $S_{5}$ & $S_{6}$ & $S_{7}$ & $S_{8}$ & $S_{9}$ & $\Phi_{1}$ & $\Phi_{2}$ & $\Phi_{3}$ \\
\hline $\mathcal{D}_{1}$ & -6 & 0 & 0 & -2 & 0 & 0 & 0 & 0 & -1 & -1 & 0 & -3 & 3 & 0 & 0 & 0 \\
$\mathcal{D}_{2}$ & 0 & 0 & 0 & -1 & 0 & 0 & 0 & 0 & -1 & -1 & 1 & -1 & 0 & 0 & 0 & 0 \\
$\mathcal{D}_{3}$ & 0 & 0 & -1 & -1 & 0 & 0 & 0 & 0 & -1 & -1 & 0 & 0 & 0 & 0 & 0 & 0 \\
$\mathcal{D}_{4}$ & 0 & 0 & 0 & 0 & 0 & 0 & 1 & 1 & -1 & -1 & 0 & 0 & 0 & 0 & 0 & 0 \\
$\mathcal{D}_{5}$ & 0 & 0 & 0 & 0 & 1 & 1 & 0 & 0 & -1 & -1 & 0 & 0 & 0 & 0 & 0 & 0 \\
$\mathcal{D}_{6}$ & 0 & -1 & 0 & 0 & 1 & 1 & 0 & 0 & -1 & -1 & 0 & 0 & 0 & 0 & 0 & 0 \\
$\mathcal{D}_{7}$ & 0 & 0 & 0 & 0 & 0 & 0 & 0 & 0 & 0 & 0 & 0 & 0 & 0 & 1 & 0 & 0 \\
$\mathcal{D}_{8}$ & 0 & 0 & 0 & 0 & 0 & 0 & 0 & 0 & 0 & 0 & 0 & 0 & 0 & 0 & 1 & 0 \\
$\mathcal{D}_{9}$ & 0 & 0 & 0 & 0 & 0 & 0 & 0 & 0 & 0 & 0 & 0 & 0 & 0 & 0 & 0 & 1 \\
\hline
\end{tabular}

